\title{
Forensic Gait Analysis and Recognition: Standards of Evidence Admissibility
}

Ioana Macoveciuc ${ }^{\mathrm{a}{ }^{*}}$, Carolyn J. Rando ${ }^{\mathrm{b}}$, and Hervé Borrion ${ }^{\mathrm{a} 2}$

${ }^{a}$ PhD Candidate, MSc; Department of Security and Crime Science, University College London, 35 Tavistock Square, London, WC1H 9EZ, United Kingdom; email: ioana.macoveciuc.16@ucl.ac.uk.

${ }^{a 2}$ Associate Professor, Director of Studies, PhD; Department of Security and Crime Science, University College London, 35 Tavistock Square, London, WC1H 9EZ, United Kingdom; email: h.borrion@ucl.ac.uk.

becturer in Bioarchaeology and Forensic Anthropology, PhD; Institute of Archaeology, University College London, 31-34 Gordon Square, London WC1H OPY, United Kingdom; email: c.rando@ucl.ac.uk.

* Corresponding author: Ms. Ioana Macoveciuc, MSc. Department of Security and Crime Science, University College London, 35 Tavistock Square, London, WC1H 9EZ, United Kingdom; email: ioana.macoveciuc.16@ucl.ac.uk. 


\begin{abstract}
Gait is one biological characteristic which has attracted strong research interest due to its potential use in human identification. Although almost two decades have passed since a forensic gait expert has testified to the identity of a perpetrator in court, the methods remain insufficiently robust for use in court, considering the recent paradigm shift witnessed in the forensic science community regarding quality of evidence. In contrast, technological advancements have taken the lead, and research into automated gait recognition has greatly surpassed forensic gait analysis in terms of the size of acquired datasets and demographic variability of participants, tested variables, and statistical evaluation of results. Despite these advantages, gait recognition presents with different problems which are yet to be resolved. Therefore, courts should treat gait evidence with caution, as they should any other form of evidence originating from disciplines without fully established codes of practice, error rates, and demonstrable applications in forensic scenarios.
\end{abstract}

KEYWORDS: forensic science, forensic gait analysis, forensic podiatry, gait recognition, evidence admissibility, standardization, cognitive bias. 
Gait, or a person's manner of walking, has long been considered an advantageous biological characteristic in human identification (1) and has been the focus of extensive research in past years, particularly due to increasing security concerns for public safety $(2,3)$. Constructed (from a physiological perspective) from a series of (largely) repetitive, cyclic movements that are generally symmetric (4), gait can be observed from a distance without the knowledge of the person of interest (5) and cannot be entirely concealed or dissimulated (6). It is therefore considered an attractive basis for developing alternative forensic identification methods since it can contribute to all stages of an investigation, including surveillance and intelligence-gathering.

The full potential of a gait-based forensic identification method is yet to be explored, despite years of research which shaped gait-based research for forensic gait analysis (7), and biometrics for gait recognition (8). Whilst forensic gait analysis and gait recognition yield a similar end-product, the methods by which analyses are performed are distinct, with the latter having demonstrated significant progress through the large number of publications to date. Forensic gait analysis uses concepts from clinical gait analysis $(7,9)$ whilst gait recognition seeks to develop person identification algorithms which may or may not have anatomical and physiological principles incorporated into their design (e.g. 10-14). However, to date, most of the gait analysis research originates from clinical and biomechanical studies which utilize state-of-the-art technologies for accurate and precise biomechanical measurements, generally intended for diagnosis and treatment of medical conditions such as stroke (15), Parkinson's disease (16), multiple sclerosis (17), and various spine disorders such as scoliosis $(18,19)$. However, data collection intended for development of forensic methods should aim to replicate a real-life scenario to obtain realistic results with quantifiable error rates, which demonstrate the utility and efficiency of the method in practice.

The principal aim of any forensic method is to provide information in the interest of justice which must abide by the rules of the relevant judicial system to be admissible as evidence in the courtroom. Since forensic science is considered a "vital instrument for the detection and deterrence of crime and the administration of justice", the standards which govern any forensic science (or science with forensic applications) must meet those imposed by the law (20). Inadequate analysis protocols offer grounds for prejudiced interpretation of the data (cognitive bias), an already recognized consequence in many forensic domains which deal with pattern matching (21) such as is the case with both gait analysis and recognition, the former also being qualitative in nature (22). The forensic science process is a balancing act between the 'raw' evidence, and the subjective, biased individual who observes and conducts tests to reach the 'truth' which, in forensic science, is provided 
in probabilistic terms, not as an absolute, objective truth; therefore, depending on the field of study in question, the levels of certainty will differ and implicitly, so will the level of confidence in the 'truth', a well-known aspect in the forensic science community. Nevertheless, the issue with lack of validation is not a criticism of the field itself or the competence of the expert, but with the implications that lack of peer-review, unvalidated methods and biased expert evidence can have on establishing guilt or innocence in court. Therefore, this paper aims to discuss the current standing of forensic gait analysis and gait recognition research in the forensic science arena, and the legal implications of applying current methods in forensic cases.

\section{The Scientific Basis of Gait in Identification}

Forensic gait analysis is defined as "the recognition and comparison of gait and features of gait, to assist the process of identification" as applied by certified podiatrists or scientists with experience in areas such as biomechanics and clinical gait analysis, who analyse two or more sets of video footage where obvious characteristics such as facial features or other personal identifiers are intentionally and/or unintentionally obscured, to conclude whether it is the same individual appearing in the videos $(23,24)$. However, in the UK, there is no official definition, accreditation process or specific regulatory body under which the 'forensic gait expert' operates and that defines the nature of the training required to allow a scientist to practice as a forensic analyst/expert. At present, forensic gait analysis is a recognized discipline of the Chartered Society of Forensic Sciences (CSFS); however, there is yet to be a regulatory group specifically designated for forensic gait analysis (although its initiation has been set forth since 2017). In addition, a code of practice produced by the CSFS for the Forensic Science Regulator is yet to be finalized to clarify these aspects (25). As a result, a variety of definitions regarding the scope and practice of forensic gait analysis are employed throughout the literature, although these should soon be superseded by the finalized code of practice definition which states that "forensic gait analysis is the analysis, comparison and evaluation of features of gait to assist the investigation of crime" (25). At present, forensic gait analysis draws methodological approaches mainly from the extensive clinical and biomechanical literature; however, the methods from such studies, although of high quality, are very different and cannot be extrapolated directly in a forensic context without adaptations necessary to function in a forensic setting and implicitly, to meet validation and peer-review requirements that are inherent to forensic practice as required by the law. The setting of a clinical laboratory intended for research contrasts that of a crime scene, and consequently, the application of gait analysis knowledge is also very different and does not consider or incorporate the challenges a forensic setting might bring, in the methodological design. 
Whereas the clinical expert observes movement from an individual both visually, and/or on a computer which displays real-time numerical data, the forensics gait expert is required to interpret gait features based on lowquality, low frame-rate CCTV footage (26-28).

When compared to clinical gait analysis, forensic gait analysis currently lacks a standardized method and criteria for analysis and interpretation, and implicitly, sufficient peer-reviewed publications to validate current methods, especially in cases where the forensic gait expert tackles poor-quality footage. Clinical gait analysis is undertaken under optimal conditions whereby, for instance, the physical 'availability' of the participant allows for repetition of movements of interest during extended periods of time, allowing for a large dataset to be built even from a single individual (e.g. 29,30). Furthermore, the clinical subject would be wearing equipment for measuring various parameters such as gait cycle duration, foot pressure values, muscular activity, and so on. This contrasts with the information available from CCTV, where factors such as total video sequence, lighting conditions, frame rate, partial obscuring of body regions, clothing, etc., significantly reduce the value of the expertise (31). Despite these known challenges, insufficient research has been conducted to date to empirically determine how these factors interact and to what extent is the expertise affected (i.e. what is the threshold for analysis to be ineffective). As a result, the expertise is currently hindered from extending beyond a 'potential confirmation' of identity or from simply 'assisting with identification', conclusions which, in fact, are based upon a highly suggestive environment where the police are already aware that the person of interest is very much likely to be the suspect they are pursuing, based upon other gathered intelligence. Such information prompts cognition to 'fill in the blanks' due to the suggestive context (contextual bias) that inadvertently leads the expert to form a prejudiced opinion (32) and therefore, interpret the data based on this opinion (confirmation bias) (33) rather than on an objective comparison between two or more videoclips.

To address one of these challenges, a CCTV footage quality assessment method was published in 2015 with the purpose of facilitating non-expert assessment of footage quality prior to investing resources in expert examination of a video which may be inadequate for forensic analysis (28). The approach was based on the Delphi Strategy that is grounded on the principle of reaching an agreed consensus between experiment participants after several rounds of testing during which each participant voices their opinion (28). In the study, the participants were asked to individually identify which 'key factors and subfactors of CCTV footage' they considered of importance for footage quality assessment based on their experience. Four key factors were identified during the first round (quality of picture, lighting, direction of footage taken relative to the suspect, 
and the suspect), with an additional one (frame rate) agreed upon after the second round. For each of these five factors, several sub-factors were added; for example, sub-factors for 'suspect' included clothing, number of steps captured and speed of movement. All the key factors with their accompanying sub-factors were then assigned a five-point Likert scale that measured the degree of agreement or disagreement of the expert with respect to which factor would impact correct analysis of CCTV footage of a forensic nature to a higher or lesser degree. The results were deemed to have 'good reliability' after having been passed on to the participating experts for testing on their own casework, who in turn reported the tool to be "fit for purpose", producing repeatable results (28). Repeatability was then tested whereby the same participating experts were asked to evaluate one CCTV videoclip once a day for five days, with the results "suggesting a good level of repeatability" (28). However, the authors of the study acknowledged that the number of experts (three experts during the first set of rounds and another two experts for an additional round) who participated in the study was too low for a Delphi Strategy approach, yet they still concluded that the results "suggest a good face validity of the tool" (28). This conclusion was also based on the positive feedback received after a trial version of the tool was made accessible to other experts for casework use. However, the feedback is vague and is unaccompanied by the case reports on which they were used, to demonstrate the utility of the tool in practice. What is further of concern is that the individualization potential of gait is taken as scientific fact despite lack of clear empirical data to demonstrate that gait is indeed unique and/or to what extent. A recent clinical study on biomechanics using human motion tracking attempts to provide some evidence regarding this aspect, concluding that forces in the foot during walking vary because gait is unique to each individual (34). However, another possible interpretation would be that this uniqueness is attributed to intra-individual variation, since the study was performed only on six subjects, all male. In the United Kingdom for example, according to Birch et al. (35), there is an ongoing development of a gait feature database (video), with roughly 1,000 individuals (59.5\% males, $40.5 \%$ females, with the majority being in the range of $18-50$ years of age and White). However, according to the authors of the publication (35), the data was obtained covertly from members of the public and all the demographic characteristics listed in the publication (available since 2016) are estimations. For more accurate assessments of inter-individual and intra-individual variation and implicitly, more accurate quantification of uncertainty levels in an expert report, known demographic variability in a population (e.g. age, sex, weight, height, body proportions (BMI)) in a dataset is evidently necessary (9). As such, at the present moment, gait analysis as performed by forensic gait experts fails to satisfy the main requirements of an identification method, which are: solid knowledge of how unique the trait used is (scientific knowledge), the 
level of variability of gait features in a given population, standardized gait features list used consistently amongst experts, appropriateness of the method forensic cases, and implementation of a code of practice for forensic gait experts. These requirements form some of the main underpinnings of what reliable expert testimony is about (36).

In contrast to forensic gait analysis, gait recognition is the automated comparison of CCTV footages to determine whether the same individual is found in the processed videoclips (37). Gait recognition research therefore differs from forensic gait analysis in that its focus is the development of algorithms which render a high recognition performance in terms of identification but also in terms of, for example, age and sex estimation of the person of interest (e.g. 38-40). With the large increase in CCTV worldwide (41-43), continuous camera monitoring is a laborious and time-consuming task due to the reduced parity between number of cameras and number of human operators. The constant demands of increased security, considering events in recent years, is likely to impact this even further and it is reasonable to assume that human identification research will continue on the route towards automatization to reduce crime and support criminal investigations, as exemplified by researchers from Osaka University, Japan, who have compiled the largest datasets yet (OU-ISIR), with 63,846 participants with known age and sex $(40,44)$. Other notable examples of datasets for gait recognition include CASIA in China (45) and SOTON in Southampton, UK (46) which include several covariates such as different clothing combinations, different environments and camera angle views but which do not contain detailed demographics such as age, as the OU-ISIR datasets do. Considering the abundance of data available, it is evident that further research will follow; also, other projects are still ongoing such as the IDENTITY project (due for completion at the end of 2019) at the University of Warwick, UK, which seeks to "consolidate the integration of multimedia forensics into the forensic sciences" by extending the use of biometrics to include gait as well (47). In addition, the UK's National Measurement Institute (NPL) together with other partners, has already developed a gait recognition system designed to track individuals and verify whether that same individual has been at the location where the original tracking took place; therefore, at present, it is at a 'verification', rather than at an 'identification' stage (48). Despite this substantial international progress in terms of database size, and covariate analysis, only a small number of studies specific for forensic application have been published to date $(2,9,10,31,49)$ including a recent pilot system with an easy-to-use user interface developed for person verification intended for users who do not have any professional training in gait analysis, such as the police force (50). As described by the authors, it is designed 
to assist in criminal investigations, whereby the user examines the list of matches presented by the system, rather than the system offering the 'correct' match (50). Whilst promising in the sense that it is the first system of this nature to be published, it was tested by a small number of participants (10 non-specialists and only one gait specialist). Demonstrating that the system is user-friendly is an important achievement of this research, as are the various databases available, in contrast with forensic gait analysis. However, a fair comparison of whether a non-specialist performs better, the same, or worse than a gait specialist, cannot be conducted using this data, a similar issue with forensic gait analysis. Also, no information is provided regarding the training of the gait specialist, their qualifications and their experience in either forensic or clinical gait analysis, therefore no conclusions can be drawn regarding differences between types and levels of training and experience. Further research is yet to be published regarding method validity, more elaborate error rates, level of uncertainty and so on, and it is unknown whether this gait recognition tool can demonstrate similar competence in, for instance, more complex scenarios (e.g. crowded shopping mall), or across populations of different ancestral background and demographic variability.

\section{The Position of Gait Analysis and Recognition in the Forensic Science Paradigm}

Cognitive biases, or unintentional errors in data interpretation due to the brain taking 'short-cuts' to reduce information processing (51), are major factors of concern widely discussed within the forensic science and legal communities (52-55). As emphasized in a 2016 report by the US President's Council of Advisors on Science and Technology (PCAST), this is of special importance in subjective, pattern-matching methods (as is forensic gait analysis), where (at present) expert judgement is heavily relied upon, in lieu of empirical data (54). Human decisions are cognitively dissonant and hence biased due to prejudices arising from professional training, work environment, experience, case context information and reference materials, and the evidence itself (56). Recommendations across a variety of forensic disciplines have been made for several years now regarding methods of reducing the subjectivity of scientific data interpretation, such as hypothesis and analysis plan formulation prior to evidence examination, reducing irrelevant case information, and peer-review, amongst many others (57-66). Forensic gait experts reportedly use one of such approaches whereby the expert first analyses the original footage, followed by the reference footage obtained of the suspect (23), thus tackling both confirmation and contextual biases using hypothesis and analysis plan formulation prior to evidence examination. However, there is little evidence in the scientific literature to demonstrate that critical evaluation of current gait analysis protocols has been conducted, despite the debates on cognitive bias and the 'correct' 
development of satisfactory protocols for collecting, analysing, and interpreting data (67). Also, only one formal case report has been published to explain and critically assess how and whether a particular protocol was effective (31).

For forensic gait analysis, an attempt was made in 2015 to develop a 'list' of recommended standardized terminology for use in practice (68). However, the 'list' is derivative from clinical gait analysis and the publication makes no clear references to how or if the definitions provided form part of an analysis protocol used in practice, despite previously raised issues about contextual bias in other pattern-matching forensic techniques such as fingerprinting (69). To date, only a small number of experimental studies have been published in forensic gait analysis which suggest good method reliability (e.g. 27,28,35,68,70), although other experts who have applied gait analysis techniques in the field refute the claim that gait-based expert evidence is sufficiently reliable for forensic use (e.g. 31, 49). However, the error rates presented in this small number of studies should be interpreted with caution because the sample number is predominantly very small, the data is not analyzed based on a standardized analysis protocol (or no explicit analysis protocol at all), and the experimental conditions are insufficiently tested for cognitive bias or other sources of error. Therefore, it is unknown whether the error rates presented are due to the training of the practitioner, inherent flaws of the analysis protocol, or whether there is an issue in the assumptions on which the method is based (54, 71-73).

At the time of writing this review, a consultation draft of a Forensic Gait analysis Code of Practice that was published in June 2018 and finalized in October, is now pending final decision (25). This draft document lists many of the challenges discussed in this review, acknowledging and providing general recommendations to cognitive bias, method validation and expert competency (25). Once complete, this should not only regulate how forensic gait analysis will be used in practice, but also guide how future research studies should be designed and evaluated. In a recent publication of the Royal Society, the UK court has also acknowledged and addressed, for the first time, the admissibility concerns of forensic gait analysis evidence. The Forensic Gait Analysis: A Primer for Courts document published in November 2017 (75), is an important step forward in strengthening the relationship between the scientist and the legal system. It not only provides an accessible, succinct and objective literature review for the court and judiciary members, but also recommends being cautious when considering gait analysis as evidence for positive identification and/or suspect exclusion, whilst considering methodological limitations (75). It also draws attention to the difficulty in assigning an objective scale of comparison between video-recordings whereby even a verbal scale of likelihood ratios would classify 
any data obtained from current methods as 'weak' (75), considering the identification percentages obtained in published studies and the absence of a large, UK population dataset. Whilst it can be debated that 'rougher' estimates of a verbal scale can be helpful to the court in the absence of large-scale population studies and that there can be high uncertainty even with large datasets (76), the problem of subjective uncertainty remains until the development of an ongoing database. As discussed in the PCAST report, case experience in pattern matching methods such as fingerprinting, cannot be a substitute for measured frequency of features in a given population (i.e. variability). The report argues that such statements made in a legal context are scientifically invalid since the absolute truth cannot be known and therefore, use of verbal scale likelihood ratios in court should be prohibited (54). Considering these aspects, the UK primer for courts is an important step in ensuring that the gap between science and the legal system is not widened, while also allowing the judiciary to make more informed decisions regarding admissibility criteria and weight of evidence from a more objective viewpoint, thus encouraging more transparent and fair trials.

In March 2018, a review article has been published in which gait experts acknowledge the challenges discussed in the primer and agree that further research into protocol design and expert training is required to ensure that methods are used and applied according to the robust standards required by forensic science and by extension, the court (77). However, there is persistence in attempting to prove that as it stands, forensic gait analysis should not fall into disrepute in the forensic science community just because it lacks these standards, and the authors appear to adopt a view that evidence should not necessarily be scientific, rather, it should be "sound" evidence (77). Considering how forensic science has changed with time, this perspective is one from which the academic community has attempted to move away $(78,79)$, precisely to improve the 'science' and by extension, to be able to provide stronger, more reliable evidence. The premise should be to empirically test methodologies, make use of appropriate statistical tests to support conclusions, continue to publish case reports which describe the utility of both new and existing methods of forensic analysis, and reflect and mitigate against cognitive biases (80-82). Whilst complete objectivity cannot be achieved throughout the entire forensic process, a higher level of objectivity is possible (83), especially if multiple hypotheses are drafted before the evidence is analyzed and the expert seeks to refute the hypotheses which they inadvertently favour (preconceived opinions), thereby helping to reduce confirmation bias (84-87). If irrelevant case information is also removed (reducing contextual bias), a stronger quality assurance protocol through peer review can be implemented, thus allowing stronger evaluation of available data to be accomplished (88). Such examples of 
approaches challenge the expert to think in ways other than those in which they are accustomed and instil a greater sense of self-critique which reduces the tendency of the brain to take cognitive 'short-cuts' and thus, reduce bias. Considering existing methods, forensic gait analysis can, at best, include or exclude an individual from a pool of individuals with similar criteria; therefore, it is still useful in guiding the police in conducting surveillance and in intelligence-gathering. At present, moving beyond this realm is challenging since variation is yet to be experimentally quantified and estimated for a given population; lack of quantifiable certainty regarding how much or how little is overall gait pattern influenced by different intrinsic variables (e.g. height, weight, ancestry, body proportions, etc.) can result in an erroneous exclusion of one or more individuals from a pool of other 'similar' individuals when in reality, the similarity may be attributed to intra-individual variation rather than to actual inter-individual differences.

In biometrics, gait is classified as a behavioural biometric (89), and the implication of this classification is that gait is not considered as individualistic as physiological biometrics (e.g. fingerprints), specifically because gait can be altered, albeit to a certain extent, by behaviour. Inebriety, emotions, state of mind and mood can all have an effect on a person's manner of walking, and implicitly, complicate the process of recognition, as do a variety of covariates such as clothing, shoes, carrying of items, type of walking surface, speed variation, amongst others (90-95). Thus, considering the large number of potential challenges which may arise, one may ask whether it is indeed worth considering gait as a biometric or even as a trait for forensic gait analysis. From a recognition viewpoint, gait is more computationally complex than other biometrics because it requires a sequence of images from video footage, instead of just one for analysis (e.g. facial recognition). However, since the gait cycle (from a recognition perspective) is composed of (generally) repetitive stages, this simplifies the process of recognition (96-98). Also, a substantial amount of research has been conducted thus far to demonstrate that such challenges can be overcome and is continuing at an even greater scale. For example, a promising avenue is being taken by a team from Osaka University in Japan who have compiled the largest gait database of video and gait energy images (whole-body shapes extracted from video) of 63,846 subjects $(31,093$ males and 32,753 females) with ages ranging from 2-90 years, the largest number of subjects being in the 615 years and 21-25 years age ranges and the lowest in the 51-90 years age range (approximately 2,000 individuals in total) (40). Other large datasets also developed by this team include treadmill datasets which comprise speed and clothing variations and gait fluctuations (92), speed transition variation dataset (93), covariate (carrying objects) dataset (94), multi-view camera angle dataset (99) and inertial sensors datasets 
$(100,101)$. Although the datasets are varied and constitute a valuable step in gait recognition research, there are several limitations to consider.

Firstly, the datasets were developed using only one type of gait recognition data format (i.e. gait energy image) which does not allow a direct comparison with other techniques available; on the corresponding webpages of the datasets, the team state that the datasets consist of one gait energy image (GEI) per participant and it is not stated whether the team is also permitted to share the video footages upon request (44). For the datasets to be used by other researchers who wish to develop new or test existing approaches other than GEI, the team would have to share the datasets in video format (although this would raise all sorts of ethical issues regarding protection of personal data, data anonymization and so on). This would be an interesting avenue to explore, considering that, to the best of the authors' knowledge, a comparison of accuracy of different biometric approaches on such a large scale has not been reported to date. In addition, the dataset only includes information on the age and sex of the participant; no information is given on ancestry, height, weight, body proportions (which have been demonstrated to provide exceedingly better results that facial features (102)), or any other particularities the participants may have. Therefore, conclusions regarding anatomical and physiological variability which are important in court, cannot be drawn.

Furthermore, the dataset is largely skewed towards younger individuals and therefore, irrespective of how large the database is as a whole, any method developed on a specific dataset, will reflect the variability observed in that dataset (in this case, the younger age groups). Since other physiological characteristics, such as height, weight and body proportions have not been recorded, conclusions regarding age-specific and sexspecific gait characteristics should be drawn with caution because the study is a cross-sectional one. For example, a younger individual and an older individual of the same sex are not directly comparable age-wise in the absence of additional, relevant data about the two individuals, because there are many other physiological factors besides age which affect gait such as body proportions and dimensions (BMI) (103-105), selection of speed $(106,107)$, and cognition $(108,109)$. Whilst the 'ideal' type of study would be a longitudinal one, feasibility of such a study remains questionable from many perspectives, including logistical and ethical. To allow for a more accurate representation of variability, researchers should aim for detailed data collection from a more uniform group of participants rather than for collecting very large datasets with skewed data towards a particular age, sex or other demographic characteristics. Similarly, the participants were filmed in an idealized environment, which is not usually the case in the real-world where, for example, a camera would have to track 
and 'extract' a particular individual from a crowd before processing the 'identification' of the individual. Hence, any gait recognition system would also have to be tested in more complex environments, to determine to what extent recognition accuracy changes, especially since many covariates have the potential to affect recognition rates and prevent recognition systems from being used in practice (110).

Lastly, potential sources of errors stemming from the algorithm design employed by the recognition system still have to be analyzed and quantified appropriately. One example of such an error source, also observed in forensic gait analysis, is that recognition technologies are not exempt from biases either and this is just one source of error that has been tackled with respect to other biometric systems such as fingerprint recognition $(111,112)$, but not fully explored. For instance, whilst completing a project on human-robot interaction several years ago, an undergraduate student at the Massachusetts Institute of Technology (MIT) discovered racial bias of a generic facial recognition algorithm which failed to recognize individuals of African-American ancestry; when interacting with the robot which was equipped with a camera and a facial recognition software, the incorporated algorithm failed to recognize the face of the student, and implicitly, her presence (113). A similar issue is present (though yet to be acknowledged research-wise) in gait recognition studies, where gait data is predominantly obtained from a small number of participants (notable exceptions are the Osaka University datasets), usually adult males of 20-35 years of age with average height and weight, with ancestral background not being reported (e.g.114-116). However, even with large datasets, no extrapolations can yet be made regarding how and to what extent are these algorithms designed to include and process human variability in different populations, how their design influences the decision-making process of the biometric system and to what extent other human cognitive biases are inadvertently introduced into their design. Some of these aspects are explicitly discussed in a document drafted by the Science and Technology Committee published by the House of Commons, UK with respect to automated face recognition, and serves as the basis for recommending "action on the governance and oversight of both forensics and biometrics... (because these) underpin essential confidence in the administration of justice" (117). Regarding gait recognition, the UK is yet to voice an opinion in the form of method validation or specific standards, since it is yet to be officially recognized as a form of identification which can be reliably used in forensic science. Considering the available codes of practice in the UK, gait recognition would currently be suited in Digital Forensics, defined in the general Code of Practice and Conduct of the Forensic Science Regulator as "the process by which information is extracted from data storage media (e.g. devices, remote storage and systems associated with computing, image, image comparison, 
video processing and enhancement (including CCTV)) ...”(118). Whilst there already is a document on method validation in digital forensics published by the Forensic Science Regulator in 2016 (119), specificity would be necessary should gait recognition evidence be used in court, since a recognition system is complex in terms of data processing and requires a trained specialist who not only should understand the system but who is also competent in analysing and interpreting data correctly and in presenting their conclusions in court together with assigning the appropriate weight to the evidence.

If biometric gait recognition is to be compared strictly with forensic gait analysis, the former is more grounded in science than the latter. Biometric studies generally make use of video recordings databases obtained under controlled conditions, are based on sound mathematics, and generally present statistically quantified recognition accuracy rates (120-122). This contrasts with forensic gait analysis which does not have a solid methodological framework or demonstrable accuracy rates and has not adequately tested whether gait experts can perform better than lay-people. Nevertheless, the collective paradigm observed in the published literature is that forensic gait analysis as conducted by gait experts, is an established forensic science, has a defined protocol, has been used in court successfully, and that the experience of the practitioner is sufficient for conducting the analysis. The negative aspects of such claims were deemed problematic by Mnookin et al (79) who discussed how forensic sciences should move toward a 'culture' founded on sound scientific principles, methodological transparency, quantifiable error rates, mitigation of cognitive bias, and continuous professional constructive criticism. In forensic gait analysis, one attempt was indeed made in 2016 to test how well lay-people can identify emotions from gait, whereby university students and members of the public $(n=30)$ were asked to analyse video files of 3 males and 3 females (70). However, this study did not include a test group of practicing forensic gait experts to determine whether experts can perform better than lay-people. Nevertheless, the authors of the publication conclude that the results "suggest" that emotions can be identified from gait, with the mention that additional research is required to understand the effects of emotions on gait and how they are actually identified (70). The accuracy rate of identification in non-forensic experts (who were knowledgeable in gait analysis) was also previously tested in 2013, where participants were asked to view video clips of 'suspect walkers' and 'target walkers' and determine whether the suspect is a match for the target walker, producing accurate matches 124 out of 175 times (123). However, there was a small number of participants $(\mathrm{n}=7)$ and the study did not include a comparison of accuracy rates with forensic gait experts. As a result, it is unknown whether forensic gait experts, due to their experience (as claimed in many studies), can 
outperform an individual with brief training in gait analysis or a lay-person for that matter. Furthermore, the lowest score was achieved by the participant who focused on the lower body (64\%), whilst the highest score was obtained by the participant who mainly used upper body features to perform the identification (80\%). However, according to the definition provided by DiMaggio and Vernon's book published in 2017, the forensic gait specialist is one who operates under the remit of podiatry (23), which mainly concerns the lower limb. However, a review published that same year concisely described that "the Forensic Podiatrist will take a hollistic view of the whole body and how movement, position, structure and function of other areas of the body affect the functioning foot, and again vice-versa" (124). Amendments to definitions, analysis methods, and interpretation strategies in light of new research are necessary to ensure good practice and should be at the core of all forensic sciences since legislations demand keeping the expert testimony within one's area of expertise (125). Nevertheless, further official clarification is required regarding the definition of forensic gait analysis, the qualifications required of the expert, and expertise limits of the gait expert.

\section{Gait evidence in court}

Over the years, the rigorousness of evidence admissibility criteria has changed at an international level, with many countries (e.g. the UK, most of the USA) now requiring analysis of evidence to be performed using validated and peer-reviewed methods with demonstrable reliabilities and error rates, by experts with appropriate qualifications $(54,126-128)$. The reason for which forensic evidence is methodologically sensitive is because it can be rendered 'unscientific' by the court (and therefore, inadmissible as evidence), if the process which was used to obtain the data does not adhere to the scientific method in a way which allows repeatability and reproducibility for validation and peer-review purposes $(129,130)$. This means that if the same protocol (repeatability) were to be applied in a similar context (reproducibility), the same levels of accuracy and precision should be achieved (80). Likewise, applying the scientific method on data which is not empirical, automatically dismisses its categorization as 'scientific data'. However, this does not imply that subjective methods are to be dismissed, but it is imperative that their limitations are clearly stated, and that they be tested to ensure validity (i.e. consistency of method across several experts) using 'black-box' studies, as recommended in the PCAST report (54). These nuances are important in court, where the accuracy of the assessment and interpretation of evidence is crucial, as it plays a leading role in the outcome of both the investigation and, where applicable, the prosecution of a suspect. Provided that the method of analysis produces accurate, unbiased evidence and conclusions are solidly based on the analyzed evidence, 
admissibility in the courtroom should not be affected; this should be irrespective of whether the discipline is classed under the forensic science umbrella or whether certain methodologies from the discipline are specifically adapted for use in the courtroom.

One reason for issues with evidence admissibility, especially in the United Kingdom, is the absence of an agreed consensus between the scientific and legal communities, an aspect also widely discussed in a 2009 report published by the US National Research Council (NAS report) addressing issues and ways to move forward in forensic science (127). As discussed in the report, there are several 'larger' matters to consider with respect to the lack of convergence between science and justice (127). Lack of research funding, structured approaches to research design, cooperation between academic and professional practice, and the constantly changing role of forensic science in general, all play an intertwined role in affecting the standing of a forensic science method in court and implicitly, evidence admissibility. This topic has also been raised over a decade ago in the UK (67) and is still being tackled, most recently with the publication of the forensic gait analysis and DNA analysis primer for courts which aim to reduce this divergence $(75,131)$. As a result of such documents published in past years, focus is now slowly being directed at the quality of the evidence itself in terms of method validity, reliability and reproducibility, rather than solely at the forensic discipline (132). This is because regardless of how 'recognized' a forensic science field is in the legal arena, this should not act as a ticket for admissibility of evidence obtained by questionable methodologies which have not been validated accordingly (133). Conversely, neither should evidence types which are less popular in the courtroom be dismissed solely on these grounds or disfavoured over expert evidence offered mostly from a 'qualifications and experience' viewpoint.

For instance, the first case to report use of expert-based gait evidence in the courtroom was that of $R v$ Saunders in 2000 in the United Kingdom. Prior to this case, gait expert evidence was an unknown in the legal arena and instead of following the systematic steps of methodological testing for validation and recognition in the peer-reviewed scientific community, forensic gait analysis appears to have become a forensic science field because of this first case in which it was applied. Nevertheless, it was not until 2011 that the admissibility of this kind of evidence was brought to a broader attention after it formed the grounds of an appeal in $R v$ Otway (23). The evidence given by the gait expert witness withstood the admissibility 'test' and was accepted despite being qualitative in nature, with no empirical support for the applied method and for the conclusions drawn. The paucity in published experimental data appears to be ignored and instead, the professional experience of 
the expert in the context of gait evidence sometimes precedes the importance of possessing relevant formal qualifications, validated methods, quantifiable error rates and assignment of appropriate weight to the evidence. By definition, an expert is one who possesses not only practical knowledge, but also the necessary qualifications which serve as validation of this knowledge, and who does not provide expert opinion on evidence which is not pertinent to their field of expertise (128). Although the UK legal system has long since dismissed the value of expert witness testimony based solely on experience (more in theory than in practice) this principle remains ambiguous in forensic disciplines such as forensic gait analysis where expert testimony given by podiatrists of clinical background using unvalidated methods has been accepted in court as 'forensically valid' evidence.

Gait analysis has also been used as evidence in court in a 2005 case in Denmark concerning a bank robbery, when the police requested this expertise because the perpetrator was disguised and they "thought that (he) had a unique gait" (31). The robbery recording was obtained via two cameras, one of which was located at the entrance to the bank and the other behind the cashier's desk. The recordings of the former were used to analyse the suspect's gait, as it gave a frontal view of the perpetrator's movements, entering, committing the crime and leaving the bank. The camera behind the desk was not placed sufficiently strategic to allow gait analysis but was used to obtain body measurements (i.e. photogrammetry technique) such as stature, eye-level height and shoulder-level height. The robbery recording was analyzed first, according to a protocol the experts devised which was based on a detailed physiological description of the entire body of the perpetrator (e.g. body angles, relative rotations of certain body regions associated with gait etc.) and on the aforementioned measurements. The experts then compared these recordings with additional recordings of a suspect obtained by the police which led them to the conclusion that there were some similarities between the two sets of recordings, which included "restless stance, anterior positioning of the head showing a neck lordosis and inversion of the ankle joint" (31). However, the robbery recording was short, the perpetrator had his hands in his pockets which created an abnormal shrugging posture, and the feet were partially obscured; nevertheless, the suspect had a limp which was concluded to be a match to that of the perpetrator, despite having based this variability on a previous study with only 11 participants. The experts concluded that besides the limp, there were insufficient characteristics for a stronger analysis to be performed, acknowledged that the analysis they conducted cannot establish the identity of the individual and stated that they did advise the court of this matter; nevertheless, the suspect was still found guilty. This is another aspect to consider when determining whether 
evidence based on unfounded scientific principles should be admissible; regardless of whether the expert outwardly declared the limitations of the method, the court still made a decision based on this evidence. In the rush to bring the guilty to justice and close a case, the court has a tendency to accept second-rated evidence in situations where no other evidence is available (i.e. the best available evidence (79)). Therefore, cooperation between the forensic scientist and the court cannot be overstated: it is necessary for the scientist to be able to refuse to perform the analysis if the method is weak and for the court to have all the necessary information (e.g. documents such as the UK primer for courts) to confer appropriate weight to the evidence (134).

\section{Conclusions}

The principles discussed in this review have become increasingly present in the scientific literature in past years in many forensic science disciplines. Criteria of admissibility of evidence in the courtroom are and should remain complex, especially in cases pertinent to new technologies, methods and/or fields of study. However, this complexity should only refer to the rigour of method testing for ensuring high-quality results rather than unnecessary difficulty in the admissibility process. If one is to consider, at least in theory, the purpose of the legal system, both overcomplication and oversimplification of this process can lead to similar results of injustice. Therefore, a stronger collaboration between the legal and scientific arenas is indispensable for ensuring transparency from all involved parties, presenting high quality evidence and instructing the jury and judges appropriately to ensure fair trials $(126,127,130)$.

To successfully meet these evidential criteria and ultimately achieve their intended purpose, the forensic sciences should work towards stronger cooperation in planning and formulating quality assurance protocols, develop and/or expand existing training programs, and strengthen collaboration between the scientific and legal communities to improve information exchange, matters discussed (amongst many others) in various documents published over the years in the US and UK (e.g. 54,55,116,117,125-128). In the case of gait analysis and recognition, a collaboration should be established between the gait analyst and the biometric specialist, where the analyst inputs their own expertise to assess the match concluded by the biometric system and also advise (from a clinical and forensic perspective) on the data collection procedures and algorithm development. The concern, as with any forensic science, is not that there is not 'enough' research being done per se, but rather how it is done (79). More so, cognitive biases and their potential pitfalls have only been briefly addressed to date. However, with the publication of the primer and of the code of practice (soon to be finalized), the standing of forensic gait analysis (in the UK at least), seems to be moving in a positive direction from both 
legal and scientific perspectives. Similarly, gait recognition is receiving increasing research attention, one example being the IDENTITY project at the University of Warwick (47). Until finalization of the forensic gait analysis code of practice and official acknowledgement of gait recognition as an identification method (together with methodological standardization), courts should remain cautious when accepting gait evidence at present, as they should be with any type of evidence which has been obtained using methods which are yet to be tested (and with quantifiable error rates), regulated by a specific code of practice and demonstrably applicable in forensic contexts.

\section{References}

(1) Kozlowski LT, Cutting JE. Recognizing the sex of a walker from a dynamic point-light display. Percept Psychophys 1977; 21(6):575-580.

(2) Condell K, Chaurasia P, Connolly J, Yogarajah P, Prasad G, Monaghan R. Automatic gait recognition and its potential role in counterterrorism. Studies in Conflict and Terrorism 2018;41(2):151-168.

(3) Home Office. Surveillance camera code of practice. London: The Stationery Office, June 2013. https://assets.publishing.service.gov.uk/government/uploads/system/uploads/attachment_data/file/204775/Su rveillance_Camera_Code_of_Practice_WEB.pdf (accessed [18 January 2019]).

(4) Patterson, K.K., Kadkarni, N.K., Black, S.E. and McIlroy, W.E. 2012. Gait symmetry and velocity differ in their relationship to age. Gait Posture 2012; 35(4):590-594.

(5) Boulgouris NV, Chi ZX. Human gait recognition based on matching of body components. Pattern Recognit 2007; 40:1763-1770.

(6) Bouchrika I, Goffredo M, Carter J, Nixon M. On using gait in forensic biometrics. J Forensic Sci 2011; 56(4):882-889.

(7) Krishan K, Kanchan T, DiMaggio JA. Emergence of forensic podiatry - a novel sub-discipline of forensic sciences. Forensic Sci Int 2015; 255:16-27.

(8) Nixon MS, Carter JN, Shutler JD, Grant MG. Automatic recognition by gait: progress and prospects. Sensor Review 2003; 23(4):323-331.

(9) Ludwig O, Dillinger S, Marschall F. Intra-individual gait pattern variability in specific situations: implications for forensic gait analysis. Forensic Sci Int 2016; 264:15-23. 
(10) Lu W, Zong W, Xing W, Bao E. Gait recognition based on joint distribution of motion angles. JCLV $2014 ; 25: 754-763$.

(11) Ariyanto G, Nixon MS. Marionette mass-spring model for 3D gait biometrics. 5th IAPR ICB; 2012 Mar 29-Apr 1; New Delhi. 2012;354-359.

(12) Liu Z, Sarkar S. Simplest representation yet for gait recognition: averaged silhouette. Proceedings of the $17^{\text {th }}$ International Conference on Pattern Recognition; 2004 Aug 26; Cambridge, UK:IEEE 2004;4:211-214.

(13) Wang C, Zhang J, Pu J, Yuan X, Wang L. Chrono-gait image: a novel temporal template for gait recognition. In: Daniilidis K, Maragos P, Paragios N, editors. Computer Vision - ECCV 2010; Lecture Notes in Computer Science 2010;6311:257-270.

(14) Sandau M, Heimburger RV, Jensen KE, Moeslund TB, Aanaes H, Alkjaer T et al. Reliable gait recognition using 3D reconstruction and random forests - an anthropometric approach. J Forensic Sci 2016;61(3):637-648.

(15) Reissman ME, Gordon KE, Dhaher YY. Manipulating post-stroke gait: exploiting aberrant kinematics. J Biomech 2018;67:129-136.

(16) Rennie L, Lofgren N, Moe-Nilssen R, Opheim A, Espen D, Franzen E. The reliability of gait variability measures for individuals with Parkinson's disease and healthy older adults - the effect of gait speed. Gait Posture 2018; 62:505-509.

(17) Roeing KL, Wajda DA, Motl RW, Sosnoff JJ. Gait termination in individuals with multiple sclerosis. Gait Posture 2015;42(3):335-339.

(18) Vergari C, Ribes G, Aubert B, Adam C, Miladi L, Ilharreborde B et al. Evaluation of a patient-specific finite element model to simulate conservative treatment in adolescent idiopathic scoliosis. Spine Deformity $2015 ; 3: 4-11$.

(19) Syczewska M, Graff K, Kalinowska M, Szczerbik E, Domaniecki J. Influence of the structural deformity of the spine on the gait pathology in scoliotic patients. Gait Posture 2012; 35(2):209-213.

(20) House of Commons Science and Technology Committee. Forensic Science on Trial. London: The Stationery Office Limited, March 2005. Seventh Report of Session 2004/5. https://publications.parliament.uk/pa/cm200405/cmselect/cmsctech/96/96i.pdf (accessed [18 January 2019]). 
(21) Dror IE, Charlton D. Why experts make errors. J Forensic Identif 2006; 56(4):600-616.

(22) Forensic Science Regulator. Forensic image comparison and interpretation evidence: guidance for prosecutors and investigators.2016;2:1-17.

https://assets.publishing.service.gov.uk/government/uploads/system/uploads/attachment_data/file/511168/Im age_Comparison_and_Interpretation_Guidance_Issue_2.pdf (accessed [18 January 2019]).

(23) DiMaggio JA, Vernon DW. Forensic gait analysis. In: DiMaggio JA, Vernon DW. Forensic Podiatry: Principles and Methods. 2nd edition. Boca Raton: CRC Press, 2017;153-180.

(24) Vernon W. The development and practice of forensic podiatry. J Clin Forensic Med 2006; 13(6-8):284287.

(25) Forensic Science Regulator. Draft Forensic Gait Analysis: Code of Practice. June 2018.

https://assets.publishing.service.gov.uk/government/uploads/system/uploads/attachment_data/file/720883/20

18_Forensic_Gait_Analysis_Consultation_Draft.pdf (accessed [18 January 2019]).

(26) Garrie DB, Morrissy DJ. Digital forensic evidence in the courtroom: understanding content and quality. Nw J Tech \& Intell Prop 2014; 12(2):121-128.

(27) Birch I, Vernon W, Burrow G, Walker J. The effect of frame rate on the ability of experienced gait analysts to identify characteristics of gait from closed circuit television footage. Sci Justice 2014; 54(2):159163.

(28) Birch I, Vernon W, Walker J, Saxelby J. The development of a tool for assessing the quality of closedcircuit camera footage for use in forensic gait analysis. J Forensic Leg Med 2013; 20(7):915-917.

(29) Hollman JH, Watkins MK, Imhoff AC, Braun CE, Akervik KA, Ness DK. A comparison of variability in spatiotemporal gait parameters between treadmill and overground walking conditions. Gait Posture 2016; 43:204-209.

(30) Josinski H, Switonski A, Jedrasiak K, Kostrzewa D. Human identification based on gait motion capture data. Proceedings of the International Multiconference of Engineers and Computer Scientists (IMEC); 2012 Mar 14-16; Hong Kong: IMED, 2012.

(31) Larsen PK, Simonsen EB, Lynnerup N. Gait analysis in forensic medicine. J Forensic Sci 2008; 53(5):1149-1153. 
(32) Edmond G, Tangen JM, Searston RA, Dror IE. Contextual bias and cross-contamination in the forensic sciences: the corrosive implications for investigations, plea bargains, trials, and appeals. Law Probability \& Risk 2015;14:1-25.

(33) Kassin SM, Dror IE, Kukucka J. The forensic confirmation bias: problems, perspectives, and proposed solutions. JARMAC 2013;2:42-52.

(34) Amirudin AN, Parasuraman S, Kadirvel A, Ahmed Khan MKA, Elamvazuthi I. Biomechanics of hip, knee, and ankle joint loading during ascent and descent walking. Procedia Comput Sci 2014; 42:336-344.

(35) Birch I, Gwinnett C, Walker J. Aiding the interpretation of forensic gait analysis: Development of a features of gait database. Sci Justice 2016; 56(6):426-430.

(36) Risinger DM. The irrelevance, and central relevance of the boundary between science and non-science in the evaluation of expert witness reliability. Villanova Law Review 2007; 52(4):679-722.

(37) Yoo JH, Hwang D, Nixon MS. Gender classification in human gait using support vector machine. In: Blanc-Talon J, Philips W, Popescu D, Scheunders P, editors. Advanced concept for intelligence vision systems. ACVIS 2005. Lecture Notes in Computer Science 2015;3708:138-145.

(38) Makihara Y, Okumura M, Iwama H, Yagi Y. Gait-based age estimation using a whole-generation gait database. International Joint Conference on Biometrics; 2011 Oct 11-13; Washington, DC. IJCB 2011;1-6. (39) Xu C, Makihara Y, Ogi G, Li X, Yagi Y, Lu J. 2017. OU-ISIR gait database comprising the large population dataset with age and performance evaluation of age estimation. IPSJ Trans CVA 2017;9:1-14.

(40) British Security Industry Association. The picture is not clear how many CCTV surveillance cameras in the UK?. July 2013. https://www.bsia.co.uk/Portals/4/Publications/195-cctv-stats-preview-copy.pdf (accessed [18 January 2019]).

(41) Surveillance Camera Commissioner. National surveillance camera strategy for England and Wales. March 2017.https://assets.publishing.service.gov.uk/government/uploads/system/uploads/attachment_data/file/6088 18/NSCS_Strategy_post_consultation.pdf (accessed [18 January 2019]). 
(42) Surveillance Camera Commissioner's IFSEC Speech. International Fire and Security Exhibition Conference; June 2016. London. https://www.gov.uk/government/speeches/surveillance-cameracommissioners-ifsec-speech (accessed [18 January 2019]).

(43) Osaka University. Yagi Laboratory. OU-ISIR Biometric Database. http://www.am.sanken.osakau.ac.jp/BiometricDB/index.html. (accessed [18 January 2019]).

(44) Centre for Biometrics and Security Research, Institute of Automation Chinese Academy of Science. CASIA Gait Database. http://www.cbsr.ia.ac.cn/english/Gait\%20Databases.asp. (accessed [18 January 2019]).

(45) Seely RD, Samangooei S, Lee M, Carter JN, Nixon MS. The University of Southampton Multibiometric tunnel and introducing a novel 3D gait dataset. IEEE Second International Conference on Biometrics: Theory, Applications and Systems; 2008 Sep 29-Oct 1; Arlington, VA. IEEE:2008;1-6.

(46) University of Warwick. IDENTITY. Project Overview. https://warwick.ac.uk/fac/sci/dcs/research/df/identity/projectoverview/ (accessed [18 January 2019]).

(47) National Physical Laboratory. Walk this way. http://www.npl.co.uk/news/walk-this-way (accessed [18 January 2019]).

(48) Lynnerup N, Larsen, PK. Gait as evidence. IET Biometrics 2014;3(2):47-54.

(49) Iwama H, Muramatsu D, Makihara Y, Yagi Y. Gait verification system for criminal investigation. IPSJ Trans CVA 2013;5:163-175.

(50) Stammers S, Bunn, S. 2015. Unintentional bias in forensic investigation. House of Parliament, POSTBRIEF. 2015;15:1-6. https://researchbriefings.parliament.uk/ResearchBriefing/Summary/POST-PB0015\#fullreport (accessed [18 January 2019]).

(51) House of Parliament. Unintentional bias in court. Parliamentary Office of Science and Technology. POSTNOTE 2015;512:1-6. https://researchbriefings.parliament.uk/ResearchBriefing/Summary/POST-PN0512\#fullreport (accessed [18 January 2019]).

(52) Risinger DM. Whose fault? - Daubert, the NAS reports and the notion of error in forensic science. Fordham Urban Law J 2010; 38(2):519-545. 
(53) Report to the President. Forensic science in criminal courts: ensuring scientific validity of featurecomparison methods. Executive Office of the President. President's Council of Advisors on Science and Technology. September 2016.

https://obamawhitehouse.archives.gov/sites/default/files/microsites/ostp/PCAST/pcast_forensic_science_rep ort_final.pdf (accessed [18 January 2019]).

(54) Forensic Science Regulator. Cognitive bias effects relevant to forensic science examinations. October 2015.https://assets.publishing.service.gov.uk/government/uploads/system/uploads/attachment_data/file/5101 47/217_FSR-G-217_Cognitive_bias_appendix.pdf (accessed [18 January 2019]).

(55) Zapf PA, Dror IE. Understanding and Mitigating Bias in Forensic Evaluation: Lessons from Forensic Science. Int J Forensic Mental Health 2017; 16(3), 227-238.

(56) Tripplett M. Errors in forensics: cause(s) and solutions. J Appl Res Mem Cog 2013; 2:63-64.

(57) Champod C. Research focused on bias will paralyse forensic science. Sci Justice 2014; 54:107-109.

(58) Elaad E. Psychological confirmation in forensic decisions. J Appl Res Mem Cog 2013; 2:76-77.

(59) Haber RN, Haber L. The culture of science: bias and forensic evidence. J Appl Res Mem Cog 2013;2:65-67.

(60) Stevenage SV, Bennett A. A biased opinion: demonstration of cognitive bias on a fingerprint matching task through knowledge of DNA test results. Forensic Sci Int 2017; 276:93-103.

(61) Mattijssen EJAT, Kerkhoff W, Berger CEH, Dror IE, Stoel RD. Implementing context information management in forensic casework: minimizing contextual bias in firearms examination. Sci Justice 2016; $56: 113-122$.

(62) Dror IE, Hampikian G. Subjectivity and bias in forensic DNA mixture interpretation. Sci Justice 2011; 51:204-208.

(63) Heyer R, Semmler C. 2013. Forensic confirmation bias: the case of facial image comparison. J Appl Res Mem Cog 2013; 2:68-70.

(64) Cole SA. Implementing counter-measures against confirmation bias in forensic science. J Appl Res Mem Cog 2013; 2:61-62. 
(65) Roberts P. Making forensic science fit for justice. Aust J Forensic Sci 2017; 49:5, 502-525.

(66) Mennell J, Shaw I. The future of forensic and crime scene science. Part I. A UK forensic science user and provider perspective. Forensic Sci Int 2006; 157S:S7-S12.

(67) Birch I, Vernon W, Walker J, Young M. Terminology and forensic gait analysis. Sci Justice 2015; 55(5):279-284

(68) Dror IE, Mnookin JL. The use of technology in human expert domains: challenges and risks arising from the use of automated fingerprint identification systems in forensic science. Probability and Risk 2010;9(1):47-67.

(69) Birch I, Birch T, Bray D. The identification of emotions from gait. Sci Justice 2016;56(5):351-356.

(70) Kassin SM, Dror IE, Kukucka J. The forensic confirmation bias: problems, perspectives, and proposed solutions. J Appl Res Mem Cog 2013; 2(1):42-52.

(71) Martire KA, Kemp RI. Considerations when designing human performance tests in the forensic sciences. Aust J Forensic Sci 2018; 50(2)166-182.

(72) Dror IE. The ambition to be scientific: human expert performance and objectivity. Sci Justice 2013; 53, $81-82$.

(73) OSAC Human Factors Committee. Draft guidance on testing the performance of forensic examiners. May 2018. https://www.nist.gov/sites/default/files/documents/2018/05/21/draft_hfc_guidance_documentmay_8.pdf (accessed (18 January 2019]).

(74) The Royal Society. Forensic Gait Analysis - A Primer for Courts. November 2017. The Royal Society. https://royalsociety.org/ /media/about-us/programmes/science-and-law/royal-society-forensic-gait-analysisprimer-for-courts.pdf (accessed [18 January 2019]).

(75) Norgaard A, Rasmusson B. The likelihood ratio as value of evidence - more than a question of numbers. Law Prob \& Risk 2012; 11:303-315.

(76) Nirenberg M, Vernon W, Birch I. A review of the historical use and criticisms of gait analysis evidence. Sci Justice 2018; 58(4):292-298. 
(77) Morgan RM. Conceptualizing forensic science and forensic reconstruction. Part I: A conceptual model. Sci Justice 2017;57(6):455-459.

(78) Mnookin JL, Cole SA, Dror IE, Fisher BAJ, Houck MM, Inman K et al. The need for a research culture in the forensic sciences. UCLA Review 2011; 58:725-779.

(79) Dror IE, Cole SA. The vision in "blind" justice: expert perception, judgement, and visual cognition in forensic pattern recognition. Psychon Bull Rev 2010; 17(2):161-167.

(80) Morgan RM. Conceptualizing forensic science and forensic reconstruction. Part II: The critical interaction between research, policy/law and practice. Sci Justice 2017;57(6):460-467.

(81) Dror IE. Human expert performance in forensic decision making: seven different sources of bias. Aust $\mathbf{J}$ Forensic Sci 2017; 49(5):541-547.

(82) Heuer RJ. Biases in evaluation of evidence. In: Heuer RJ. Psychology of intelligence analysis. Central Intelligence Agency. 1999;115-126.

(83) Dror IE, Charlton D, Peron AE. Contextual information renders experts vulnerable to making erroneous identification. Forensic Sci Int 2006; 156:74-78.

(84) Simon D, Pham LB, Le QA, Holyoak KJ. The emergence of coherence over the course of decision making. J Exp Psychol Learn Mem Cogn 2001; 27(5):1250-60.

(85) Heuer, RJ Jr. Analysis of competing hypotheses. In: Heuer, RJ Jr. Psychology of intelligence analysis. Centre for the study of intelligence.1999;95-110.

(86) Kukucka J, Kassin SM, Zapf PA, Dror IE. Cognitive bias and blindness: a global survey of forensic science examiners. JARMAC 2017;6(4):452-459.

(87) Ballantyne KN, Edmond G, Found B. Peer review in forensic science. Forensic Sci Int 2017;277:66-76.

(88) Chai Y, Ren J, Han W, Li H. Human gait recognition: approaches, datasets and challenges. 4th International Conference on Imaging for Crime Detection and Prevention; 2011; London. IET, 2012;1-6.

(89) Guan Y, Chang-Tsun L, Roli F. On reducing the effect of covariate factors in gait recognition: a classifier ensemble method. IEEE Trans Pattern Anal Mach Intell 2015; 37(7):1521-1528. 
(90) Yam CY, Nixon MS, Carter, JN. Automated person recognition by walking and running via modelbased approaches. Pattern Recog 2003; 37(5):1057-1072.

(91) Makihara Y, Mannami H, Tsuji A, Hossain MA, Sugiura K, Mori A et al. The OU-ISIR gait database comprising the treadmill dataset. IPSJ Trans on Computer Vision and Applications 2012;4:53-62.

(92) Mansur A, Makihara Y, Aqmar R, Yagi Y. Gait recognition under speed transition. IEEE Conference on Computer Vision and Pattern Recognition 2014 Jun 23-28. Columbus OH. IEEE, 2014:2521-2528.

(93) Uddin MZ, Ngo TT, Makihara Y, Takemura N, Li X, Muramatsu D et al. The OU-ISIR Large Population Gait Database with Real-Life Carried Object and its performance evaluation. IPSJ Trans. on Computer Vision and Applications 2018;10(1):1-5.

(94) Choudhury SD, Tjahjadi T. Clothing and carrying condition invariant gait recognition based on rotation forest. Pattern Recognit Lett 2016; 80:1-7.

(95) Schutte LM, Narayanan U, Stout, JL, Selber P, Gage JR, Schwartz MH. An index for quantifying deviations from normal gait. Gait Posture 2000;11(1):25-31.

(96) Schwartz MH, Rozumalski A. The gait deviation index: a new comprehensive index of gait pathology. Gait Posture 2008; 28(3):351-357.

(97) Polana R, Nelson R. Low level recognition of human motion (or how to get your man without finding his body parts. Proceedings of the 1994 IEEE Workshop on Motion of Non-Rigid and Articulated Objects; 1994 Nov 11-12; Austin, TX. IEEE, 2002.

(98) Takemura N, Makihara Y, Muramatsu D, Echigo T, Yagi Y. Multi-view large population gait dataset and its performance evaluation for cross-view gait recognition IPSJ Trans on Computer Vision and Applications 2018;10(4):1-14.

(99) Ngo TT, Makihara Y, Nagahara H, Mukaigawa Y, Yagi Y. The largest inertial sensor-based gait database and performance evaluation of gait-based personal authentication. Pattern Recog 2014;47(4):222231.

(100) Ngo TT, Makihara Y, Nagahara H, Mukaigawa Y, Yagi Y. Similar gait action recognition using inertial sensor. Pattern Recog 2015;48(4):1289-1301. 
(101) Lucas T, Henneberg M. Comparing the face to the body, which is better for identification?. Int J Leg Med 2016; 130(2):533-540.

(102) D’Hondt E, Deforche B, de Bourdeaudhuij I, Gentier I, Tanghe A, Shultz S, et al. Postural balance under normal and altered sensory conditions in normal-weight and overweight children. Clin Biomech 2011; 26(1):84-89.

(103) Meng H, O’Connor DP, Lee B-C, Layne CS, Gorniak SL. Alterations in over-ground walking patterns in obese and overweight adults. Gait Posture 2017; 53:145-150.

(104) Krkeljas Z. Changes in gait and posture as factors of dynamic stability during walking in pregnancy. Human Mov Sci 2018;58:315-320.

(105) Van Hamme A, El Habachi A, Samson, W, Dumas R, Cheze L, Dohin B. Gait parameters database for young children: the influences of age and walking speed. Clin Biomech 2015; 30(6):572-577.

(106) Blair S, Lake MJ, Ding R, Sterzing T. Magnitude and variability characteristics when walking on an irregular surface at different speeds. Human Mov Sci 2018; 59:112-120.

(107) Valkanova V, Esser P, Demnitz N, Sexton CE, Zsoldos E, Mahmood B et al. Association between gait and cognition in an elderly population-based sample. Gait Posture 2018; 65:240-245.

(108) Demnitz N, Hogan DB, Dawes H, Johansen-Berg H, Ebmeier KP, Poulin MJ et al. Cognition and mobility show a global association in middle- and late-adulthood: analyses from the Canadian longitudinal study on aging. Gait Posture 2018;64:238-243.

(109) Connor P, Ross A. Biometric recognition by gait: a survey of modalities and features. Comp Vis Image Underst 2018; 167:1-27.

(110) Dror IE, Mnookin JL. The use of expert technology in human expert domains: challenges and risks arising from the use of automated fingerprinting identification systems in forensic science. Law Prob \& Risk 2010; 9:47-67.

(111) Dror IE, Wertheim K, Fraser-Mackenzie F, Walajtys J. The impact of human-technology cooperation and distributed cognition in forensic science: biasing effects of AFIS contextual information on human experts. J Forensic Sci 2012;57(2):343-352. 
(112)Buolamwini J. 2017. How I'm fighting bias in algorithms. TED Talk.

https://www.media.mit.edu/posts/how-i-m-fighting-bias-in-algorithms/. (accessed [18 January 2019]).

(113) Li W, Kuo CCJ, Peng J. Gait recognition via GEI subspace projections and collaborative representation classification. Neurocomputing 2018; 275:1932-1945.

(114) Yu S, Chen H, Wang Q, Shen L, Huang, Y. Invariant feature extraction for gait recognition using only one uniform model. Neurocomputing 2017; 239:81-93.

(115) Al-Tayyan A, Assaleh K, Shanableh T. Decision-level fusion for single-view gait recognition with various carrying and clothing conditions. Image Vis Comput 2017; 61:54-69.

(116) House of Commons, Science and Technology Committee. Biometrics strategy and forensic services. House of Commons. May 2018;1-31. $5^{\text {th }}$ Report of Session 2017-2019.

https://publications.parliament.uk/pa/cm201719/cmselect/cmsctech/800/800.pdf (accessed [18 January 2019]).

(117) Forensic Science Regulator. Codes of Practice and Conduct for Forensic Science Providers and Practitioners in the Criminal Justice System. 2017;4:1-67.

https://assets.publishing.service.gov.uk/government/uploads/system/uploads/attachment_data/file/651966/10 0_-_2017_10_09_-_The_Codes_of_Practice_and_Conduct_-_Issue_4_final_web_web_pdf_2_.pdf (accessed [18 January 2019]).

(118) Forensic Science Regulator. Guidance: Method validation in digital forensics. 2016;1:1-45.

https://assets.publishing.service.gov.uk/government/uploads/system/uploads/attachment_data/file/528123/FS R_Method_Validation_in_Digital_Forensics_FSR-G-218_Issue_1.pdf (accessed [18 January 2019]).

(119) Alawar HM, Ugail H, Kamala M, Connah D. The Bradford Multi-Modal Gait Database: Gateway to Using Static Measurements to Create a Dynamic Gait Signature. Br J Appl Sci Technol 2016; 14(1):1-10.

(120) Sarkar S, Phillips PJ, Liu Z, Vega IR, Grother P, Bowyer KW. The HumanID gait challenge problem: data sets, performance and analysis. IEEE Trans Pattern Anal Mach Intell 2005; 27(2):162-177.

(121) Chen C, Liang J, Zhu X. Gait recognition based on improved dynamic Bayesian networks. Pattern Recog 2011; 44:988-995. 
(122) Birch I, Raymond L, Christou A, Fernando MA, Harrison N, Paul F. The identification of individuals by observational gait analysis using closed circuit television footage. Sci Justice 2013; 53(3):339-342.

(123) Burrow JG, Kelly HD, Francis BE. Forensic podiatry - an overview. JFSCI 2017; 5(4):1-8.

(124) Ministry of Justice. The Criminal Procedure Rules. 2018. https://www.justice.gov.uk/courts/procedurerules/criminal (accessed [18 January 2019]).

(125) Home Office. Forensic Science Strategy: A national approach to forensic science delivery in the criminal justice system. March 2016.

https://assets.publishing.service.gov.uk/government/uploads/system/uploads/attachment_data/file/506652/54 493_Cm_9217_Forensic_Science_Strategy_Accessible.pdf (accessed [18 January 2019]).

(126) National Research Council. Strengthening Forensic Science in the United States: A Path Forward. Committee on Identifying the Needs of the Forensic Sciences Community. 9The National Academies Press: Washington. August 2009. https://www.ncjrs.gov/pdffiles1/nij/grants/228091.pdf (accessed [18 January 2019]).

(127) The Crown Prosecution Service. Expert Evidence. 2014.

https://www.cps.gov.uk/sites/default/files/documents/legal_guidance/expert_evidence_first_edition_2014.pd f (accessed [18 January 2019]).

(128) O’Brien E, Daeid NN, Black S. Science in the court: pitfalls, challenges and solutions. Philos Trans 2015; 370(1674):1-7.

(129) Robertson J. Understanding how forensic science may contribute to miscarriages of justice. Aus J Forensic Sci 2013; 45(2):109-112.

(130) The Royal Society. Forensic DNA Analysis - A Primer for Courts. November 2017. The Royal Society. https://royalsociety.org/ /media/about-us/programmes/science-and-law/royal-society-forensic-dnaanalysis-primer-for-courts.pdf (accessed [18 January 2019]).

(131) Koehler JJ, Meixner JB. An empirical research agenda for the forensic sciences. J Crim Law Criminol 2016; 106(1):1-35.

(132) Evett IW, Jackson G, Lambert JA. More on the hierarchy of propositions: exploring the distinction between explanations and propositions. Sci Justice 2000;40(1):3-10. 
(133) van Asten AC. On the added value of forensic science and grand innovation challenges for the forensic community. Sci Justice 2014; 54(2):170-179. 